\title{
ANÁLISE DA PRESENÇA NO REFEITÓRIO ESCOLAR DA DIETA VEGETARIANA
}

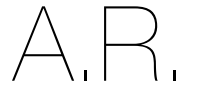
ARTIGO DE REVISÃO

${ }^{1}$ Uniself, S.A.,

Rua de S. Gens, n. ${ }^{\circ} 3380$ $\mathrm{N}, 1^{\circ}$,

4460-409 Senhora da Hora, Portugal

‘Endereço para correspondência: Isa Brandão Uniself, S.A.

Rua de S. Gens, n. ${ }^{\circ} 380, \mathrm{~N}, 1$, 4460-409 Senhora da Hora,

Portugal

brandaoimb@gmail.com

Histórico do artigo:

Recebido a 5 de dezembro de 2019 Aceite a 31 de março de 2020

\section{ANALYSIS ABOUT THE PRESENCE IN THE SCHOOL CANTEEN OF THE VEGETARIAN DIET}

Isa Brandão"; Helena Ávila

\section{RESUMO}

A alimentação é um pilar fundamental na saúde das populações, em especial na população em idade pediátrica. Este período da vida é caracterizado por um rápido crescimento e desenvolvimento físico e intelectual sendo particularmente vulnerável a desequilíbrios nutricionais que poderão ter consequências irreversíveis no crescimento e na saúde. A dieta vegetariana tem vindo a ganhar relevo na sociedade, e por isso, torna-se relevante refletir sobre o impacto desta dieta na saúde e educação das crianças e adolescentes em idade escolar. Em Portugal, a par da refeição enquadrada nos princípios da Dieta Mediterrânica, passou a ser obrigatório uma opção vegana nos refeitórios escolares. Neste sentido, parece ser importante refletir sobre os possíveis efeitos a médio e a longo prazo desta medida, designadamente o seu pretenso nivelamento com a Dieta Mediterrânica em contexto de alimentação em meio escolar.

\section{PALAVRAS-CHAVE}

Crianças, Dieta vegetariana, Nutrição Pediátrica, Refeitório escolar, Veganismo, Vegetarianismo

\section{ABSTRACT}

Feeding is a fundamental pillar in the health of populations, especially for the population in paediatric age. This period of life is characterized by rapid growth and physical and intellectual development and is particularly vulnerable to nutritional imbalances that may have irreversible consequences for growth and health. The vegetarian diet has been gaining importance in the society. Therefore, it is relevant to reflect on the impact of this diet on the health and education of school-age children and adolescents. In Portugal, along with a meal framed by the principles of the Mediterranean Diet, a vegan option in school canteens became mandatory. In this sense, it seems important to reflect on the possible medium and long term effects of this measure, namely its alleged levelling with the Mediterranean Diet in the context of school feeding.

KEYWORDS

Children, Vegetarian diet, Paediatric nutrition, School canteen, Veganism, Vegetarianism

\section{INTRODUÇÃo}

A dieta vegetariana (DV) tem vindo a ocupar um lugar especial na sociedade moderna nos últimos anos. Principalmente por questões ambientais e pela preocupação dos consumidores no direito e defesa dos animais (1). Em princípio, pais vegetarianos tendem a educar os seus filhos neste tipo de dieta (2) sendo que, em Portugal e em 2017, entrou em vigor a Lei n. ${ }^{\circ}$ 11/2017 de 17 de Abril (3) que obriga à existência de uma opção vegetariana no refeitório escolar, a par da refeição prevista nas circulares emanadas pela Direção-Geral da Educação enquadrada nos princípios da Dieta Mediterrânica (DM). Não obstante desta obrigatoriedade, pouco foi refletido sobre o impacto na saúde das crianças de uma DV e possíveis consequências educacionais. Este trabalho pretende abordar alguns assuntos relacionados com a DV, designadamente o seu pretenso nivelamento com a DM em contexto de alimentação em meio escolar.
O Vegetarianismo: Definição, Conceitos e Caracterização O padrão alimentar vegetariano utiliza predominantemente produtos de origem vegetal e exclui a carne e o pescado. A exclusão de laticínios e os ovos poderá ser total ou parcial o que faz com que o termo "vegetariana" receba uma terminologia distinta, a saber: ovolactovegetariana: permite ovos e laticínios; lactovegetariana: exclui ovos, mas permite laticínios; ovovegetariana: exclui laticínios, mas permite ovos; vegetariana estrita ou vegana: exclui todos os alimentos de origem animal (1). De notar que, apesar das definições aqui apresentadas serem as mais comuns, não são as únicas. Por exemplo, Beardsworth \& Keil (1991) desenvolveram uma escala para definir uma gama de DV consoante a sua restrição em alimentos de origem animal (de tipo 1: a menos restritiva até tipo 6: a mais restritiva) (4), Le et al. (2018) utilizam definições diferentes para estes mesmos termos, agora de acordo com a frequência de ingestão de produtos de origem 
animal (5). Phillips (2005) e Dagnelie \& Mariotti (2017) apresentam definições para outras variantes da alimentação vegetariana como a alimentação semivegetariana, pseudovegetariana, vegetarina crudívora, frutariana, macrobiótica, entre outras $(6,7)$. Não obstante a diversidade de padrões alimentares existentes, neste trabalho iremos considerar o termo vegetariano para incluir, sem distinção, todos os tipos de DV que foram adotadas de forma consciente e voluntária, a referenciar: a dieta ovolacto-, lacto-, ovovegetariana, vegetariana estrita ou vegana, a não ser que seja mencionado o contrário.

As razões que levam as pessoas a aderirem a uma DV, mesmo diversas, podem ser divididas em três grandes categorias: (i) intelectuais (éticas, morais, religiosas, espirituais); (ii) sociais (ecológicas, económicas, políticas); (iii) físicas (saúde, higiene, toxicologia, desempenho físico) (8). Estas razões têm como base a adesão resultante da sua tomada de decisão, embora se estime que milhões de pessoas em todo o mundo sigam dietas predominantemente ou exclusivamente baseadas em alimentos de origem vegetal, mas estas são vegetarianas "não-opcionais", dado os alimentos de origem animal não estarem disponíveis localmente ou serem inacessíveis. É o caso de países em subdesenvolvimento com escassez de alimentos em geral e/ou países com severas desigualdades económicas e sociais $(9,10)$. Além disso, e especialmente em países não ocidentais, o vegetarianismo pode estar relacionado com a aspetos culturais e religiosos (7). A razão principal da adoção consciente e voluntária de uma DV parece ser intelectual, nomeadamente por orientações ético-morais tendo, assim, surgido o conceito de "vegetariano ético" $(11,12)$. A segunda razão parece ser por motivos de saúde (13-15). Em Portugal, dados não oficiais de 2009 obtidos por um inquérito realizado online pelo Centro Vegetariano mostram que dos 379 inquiridos que se consideram vegetarianos, $68 \%$ assinalaram que a sua escolha se deve a motivos éticos (16).

Em geral, as crianças e adolescentes vegetarianas são-no por exemplo dos hábitos alimentares dos próprios pais, mas os adolescentes que iniciam este tipo de alimentação parecem fazê-lo principalmente por questões éticas (17).

Números oficiais da prevalência de vegetarianos no mundo e, em particular, em Portugal são escassos ou inexistentes (18). Acresce que algumas estimativas são obtidas por inquéritos não oficiais a uma amostra da população e que, nalguns questionários, as respostas são reportadas como perceção do próprio consumidor acerca da definição do termo vegetariano, e não analisando a ingestão alimentar. Assim sendo, as estimativas indicam que a percentagem de adultos vegetarianos encontra-se nos 20-30\% em Indianos (19-21), 14\% em Brasileiros (22), 11,2\% em Australianos (23), 10\% em Alemães (24), 7\% em Britânicos (25), 5\% em Chineses (26), 3,7\% em Americanos (27), 1,3\% em Espanhóis (28) e 1,2\% em Portugueses (29). A maioria dos dados relatados é limitada à população adulta e há pouca informação disponível sobre o uso de dietas vegetarianas e veganas (DV) em crianças e adolescentes. Num inquérito nacional sobre consumo alimentar realizado na Alemanha entre 2003 e 2006, cerca de 2,1\% dos rapazes e $6,1 \%$ das raparigas com idades entre 14 - 17 anos seguiam uma DV (30). Em 2015, 15\% dos jovens alemães (16 - 24 anos) consideravam-se vegetarianos (31). Segundo uma investigação realizada a 1500 crianças britânicas de idades entre 8 - 16 anos, por Linda McCartney Foods, 10\% das crianças identificaram-se como vegetarianas (32). Em 2014, um inquérito realizado online pela Harris Poll a 1213 crianças entre os 8 e 18 anos de idade, nos Estados Unidos da América, 4\% mencionam nunca comer carne e desses 1\% são veganos (33). Em Portugal, não existem dados sobre a prevalência de crianças vegetarianas, mas é possível que pais vegetarianos eduquem os filhos neste tipo de dieta (2).

\section{O Vegetarianismo e a Saúde}

Até ao momento, não existe uma posição unânime entre as associações internacionais de nutrição sobre DV nos jovens, como seguidamente se demonstra. Por um lado, as posições da American Academy of Pediatrics (AAP, 2013) (34) e da Academy of Nutrition and Dietetics (AND, 2016) (35) parecem ser consensuais. A AND (2016) menciona que a DV, incluindo a vegana, se devidamente planeada, poderá ser implementada em todas as fases da vida (35). Posição semelhante é tomada pelo National Health and Medical Research Council Australiano no seu guia alimentar de 2013 (36), dando especial atenção à dieta vegana. Este guia menciona que é importante serem atingidas as necessidades energéticas diárias, que o consumo de alimentos vegetais seja variado, de modo a garantir uma ingestão adequada de ferro (Fe), zinco (Zn) e cálcio (Ca) e que pode ser necessária a suplementação de vitamina B12 (B12). O grupo de trabalho da Sociedade Italiana de Nutrição Humana argumenta que "as DV bem planeadas, que incluam uma ampla variedade de alimentos vegetais e uma fonte segura de B12, fornecem uma ingestão adequada de nutrientes", no entanto, é necessário que "o governo e organizações de saúde / nutrição forneçam mais e melhor informação para ajudar os italianos a consumir uma DV nutricionalmente adequada" (37). No Brasil, um parecer do Conselho Regional de Nutricionistas 3. ${ }^{a}$ Região toma a posição do AND (2016) (38). A Canadian Pediatric Society (CPS, 2010) considera que uma DV equilibrada pode atender às necessidades de crianças e adolescentes, no entanto, uma ingestão calórica adequada deve ser assegurada e o crescimento monitorizado (39). Por outro lado, a Associação Alemã de Nutrição (Deutsche Gesellschaft für Ernährung) não recomenda uma dieta vegana na "gravidez, lactação, infância e adolescência", devido a um elevado risco de deficiências nutricionais, especialmente a B12 (40). Em 2017 , a European Society for Paediatric Gastroenterology, Hepatology, and Nutrition (ESPGHAN) na sua posição sobre alimentação complementar (41) menciona que "as dietas veganas geralmente são desencorajadas durante a alimentação complementar. Embora, teoricamente, uma dieta vegana possa atender aos requisitos nutricionais quando a mãe e o bebé seguem conselhos médicos e nutricionais em relação à suplementação, os riscos de não seguir os conselhos são graves, incluindo danos cognitivos irreversíveis por deficiência de vitamina B12 e morte" (42).

Em Portugal, a Direção-Geral da Saúde (DGS), no âmbito do Programa Nacional para a Promoção da Alimentação Saudável (PNPAS), lançou três manuais sobre vegetarianismo, um com linhas de orientação sobre alimentação vegetariana saudável (43), outro sobre alimentação vegetariana em idade escolar (1) e outro sobre planeamento de refeições vegetarianas para crianças em restauração coletiva (44). Estes manuais foram criados com o objetivo de contribuir para um maior conhecimento dos profissionais de saúde e da população em geral sobre DV e não uma forma de promover a DV (45). Nos três manuais são subscritas as posições americanas, nomeadamente a da AND (2016). Tanto a DGS como a Ordem dos Nutricionistas acautelam os riscos para uma alimentação vegetariana, em particular a estrita, e a necessidade de acompanhamento por um nutricionista (46). Note-se

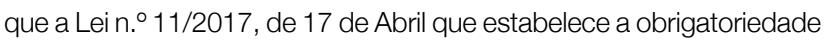
de existência de opção vegetariana nas ementas das cantinas e refeitórios públicos, determina que a mesma seja vegetariana estrita, não admitindo uma opção ovolactovegetariana (47).

Não serão de estranhar as diferentes posições e respetivos alertas das entidades internacionais e nacionais mencionadas. A idade pediátrica que, em Portugal, se estende dos 0 até aos 18 anos de idade (Despacho n. ${ }^{\circ} 9871 / 2010$ de 1 de Junho), é um período de grande vulnerabilidade 
a alterações nutricionais (1). Durante a infância e adolescência ocorre um rápido crescimento físico e desenvolvimento cognitivo que leva a que as necessidades em macro e micronutrientes estejam estabelecidas. Assim sendo, uma DV deverá seguir os princípios de um regime saudável, isto é, completa, variada e equilibrada (48). A DV apresenta um padrão de restrição alimentar, com a exclusão completa de qualquer produto de origem animal. De facto, o risco de carência nutricional inerente a este tipo de alimentação, quando mal planeado, é elevado com possíveis consequências graves no desenvolvimento normal da criança $(1,49)$. Num estudo realizado a adolescentes que seguiram uma dieta vegana até aos 6 anos de idade, mesmo depois de passarem para dietas menos restritivas, aqueles apresentavam pontuações mais baixas em testes de medição das capacidades cognitivas do que os adolescentes com uma dieta omnívora desde a nascença (50). Além disso, o artigo de revisão mais recente, realizado em 2017, que pretendeu avaliar o estado nutricional ou de saúde de crianças e adolescentes vegetarianos (0-18 anos) mostra que, na generalidade, as crianças veganas estão no percentil mais baixo do peso e altura, e têm um maior risco de deficiência de ferro (51).

Conhecendo estes riscos para a saúde, alguns autores e instituições de saúde pública lançaram publicações científicas com recomendações e materiais para os profissionais da saúde para elaboração de uma dieta vegetariana planeada na infância (por exemplo, (49)).

\section{A Dieta Mediterrânica}

A Dieta ${ }^{1}$ Mediterrânica foi reconhecida em 2013 pela UNESCO como Património Cultural Imaterial da Humanidade de Itália, Portugal, Espanha, Marrocos, Grécia, Chipre e Croácia (52). Trata-se de um modelo cultural, histórico e de saúde que deverá ser preservada (53) e promovida (54). O conceito de DM integra um padrão alimentar muito além do consumo de per si, refletindo um modo de viver evolutivo e adaptado a um determinado contexto ambiental, social, cultural e religioso (54). A DM é caracterizada pela abundância de alimentos de origem vegetal (cereais integrais ou pouco refinados, produtos hortícolas, fruta, leguminosas secas e frescas e frutos secos e oleaginosos); pela utilização de produtos frescos, pouco processados e locais, respeitando a sua sazonalidade; pela utilização do azeite como principal fonte de gordura; pelo consumo frequente de pescado; pelo consumo baixo a moderado de laticínios; pelo consumo de pequenas quantidades de carnes vermelhas e ingestão moderada de vinho, geralmente durante as refeições; pelo consumo de água (1,5L a $2 \mathrm{~L}$ por dia) como a bebida de eleição; por confeções culinárias simples e com os ingredientes nas proporções certas. A prática diária de exercício físico moderado está presente neste modo de vida (55). Esta dieta apresenta inúmeros benefícios para a saúde, longevidade e qualidade de vida, em princípio, pelo seu baixo teor de AG saturados, alto teor de AG insaturados, vitaminas, minerais, antioxidantes e fibra $(56,57)$. Dinu et al. (2017) investigaram o impacto da DM em 37 resultados na saúde, incluindo mortalidade geral, cancro, doenças cardiovasculares, entre outros. O estudo consistiu numa análise feita em 13 meta-análises de estudos observacionais e 16 meta-análises de estudos randomizados controlados, com um total de doze milhões e oitocentas mil pessoas envolvidas. Concluíram que a adesão à DM reduziu o risco de mortalidade geral, diminui a incidência e mortalidade por doenças cardiovasculares, diminuiu a incidência de doença coronária, diminuiu o número de eventos cardiovasculares como enfartes do miocárdio e acidentes vasculares cerebrais, diminuiu a incidência e mortalidade por cancro em geral,

'do grego "daiata", que significa modo de vida (in: Falcato J, Graça P. A Evolução Etimológica e Cultural do Termo "Dieta". Revista Nutrícias [Internet]. 2015(24):12-5.) diminuição do aparecimento de doenças neurodegenerativas e da diabetes. Também mostraram que a DM é extremamente eficaz na redução de peso, do perímetro abdominal, na redução do colesterol e melhoria dos níveis do HDL, quando comparada com dietas controlo (58). Outros estudos mostram que a DM parece ser eficaz na redução do risco de fratura (59) e no aumento de longevidade (60).

Tilman \& Clark (2014) analisaram as relações entre diversas dietas e a saúde e sustentabilidade ambiental. Mostram que diferentes dietas poderão ter maior impacto na diminuição do risco de doença específica, por exemplo, a DV com a diminuição do risco de diabetes tipo 2 e a DM com a diminuição da mortalidade por doença coronária e mortalidade geral, em comparação com uma dieta omnívora "convencional" (61). No entanto, verifica-se que a DV não tem impacto na mortalidade geral (62-65), estando este benefício bem demonstrado para a DM, como mencionado anteriormente.

Por estes motivos, não é de estranhar que a DM seja promovida no âmbito de uma alimentação saudável nas escolas (54). Em Portugal, a adesão ao padrão alimentar Mediterrânico (PAM) foi avaliada para a população adulta, através da análise efetuada aos dados obtidos do Inquérito Alimentar Nacional e de Atividade Física (IAN-AF) realizado entre 2015-2016. No relatório publicado em 2017, três níveis de adesão ao PAM foram definidos de acordo com o Mediterranean Diet Score e, mostra que, 31\% dos indivíduos apresenta baixa adesão ao PAM, 50\% dos indivíduos apresenta adesão moderada e, apenas 18\% dos indivíduos apresenta adesão elevada ao PAM. Além disso, os indivíduos com insegurança alimentar moderada/grave apresentam menor prevalência de elevada adesão ao PAM (66) como é constatado noutros estudos (67). Pereira-da-Silva et al. (2016), com o objetivo de analisar a dieta de crianças no pré-escolar nos países mediterrânicos da União Europeia, verificaram que a maioria das crianças que vivem nos países analisados têm uma baixa adesão a uma DM. Esta baixa adesão foi associada ao excesso de peso e obesidade nas crianças. Além disso, o consumo de dietas menos saudáveis pelas crianças estava associado com um menor estatuto socioeconómico dos pais (68). Um estudo mais recente realizado a crianças e adolescentes mostra resultados semelhantes (69). Vários autores alertam sobre a crise de excesso de peso e obesidade nas crianças a nível mundial e a necessidade de existência de políticas de saúde que invistam fortemente na disseminação dos princípios da DM como um modelo a seguir (70), não só através de informação mas também da alteração de ambientes e trabalhando as desigualdades socioeconómicas e a insegurança alimentar $(71,72)$.

\section{Alimentação em Meio Escolar}

Em 2006, a Organização Mundial da Saúde (OMS) alertava para a necessidade das políticas nutricionais e alimentares nas escolas prevenirem ou reverterem os efeitos adversos na saúde da crescente prevalência do excesso de peso e de obesidade e maus hábitos alimentares nas crianças e adolescentes (73). Em 2007, a Comissão das Comunidades Europeias, em linha com a OMS, ressalvava a importância das escolas na educação das crianças em estilos de vida saudáveis como a alimentação e a prática de exercício físico (74). Em Portugal, desde os anos 80 do século XX que o Ministério da Educação se vem preocupando com as questões da alimentação (72). De facto, alguns exemplos de programas de oferta alimentar em meio escolar incluem a distribuição gratuita de leite (75), distribuição gratuita de frutas e hortícolas (76), os géneros alimentícios (GA) que se encontram disponíveis no bufete e máquinas de venda automática (77). O Ministério da Educação e Ciência tem levado a cabo várias iniciativas com vista à melhoria nutricional das refeições servidas nas escolas, visíveis nos seguintes documentos: “Educação Alimentar em 
Meio Escolar Referencial para uma Oferta Alimentar Saudável" (DGIDC, 2006) (78), "Ofício Circular n. ${ }^{\circ}$ 7/DGE/2012 - Bufetes Escolares - Orientações" (77), "Circular n. 3097/DGE/2018 - Orientações sobre Ementas e Refeitórios Escolares" (79). Nesta última, é clara a promoção da DM, ao mencionar que a Direção-Geral da Educação (DGE), "como membro integrante do Grupo de Acompanhamento para a salvaguarda e promoção da Dieta Mediterrânica (GADM) mas também como responsável pelas orientações sobre a oferta alimentar em meio escolar, tem como missão a promoção junto das escolas, a sua divulgação e defesa desta saudável forma de alimentação (...) devendo a elaboração das ementas contemplar os princípios da Dieta Mediterrânica". Referem ainda que as refeições escolares, além de providenciarem a satisfação das necessidades energéticas e nutricionais das crianças, possuem outros objetivos, nomeadamente pedagógicos, sociais, ambientais, culturais e de saúde $(54,79)$.

\section{Alimentação Vegetariana em Meio Escolar}

Em Março de 2016, deu entrada na Assembleia da República uma petição pública pela "inclusão de opções vegetarianas nas escolas, universidades e hospitais portugueses" criada pela Associação Vegetariana Portuguesa, tendo sido obtidas mais de 15000 assinaturas (80). Um projeto de lei deu seguimento a esta petição, o projeto de Lei n. ${ }^{\circ} 111 / X I I I / 1{ }^{a}{ }^{a}$ "Inclusão de opção vegetariana em todas as cantinas públicas", que foi aprovado a 3 de março de 2017 e assim foi criada a Lei n. ${ }^{\circ}$ 11/2017 de 17 de Abril que estabelece a obrigatoriedade de existência de uma opção vegetariana nas ementas das cantinas e refeitórios públicos (mais precisamente, vegana) (3).

Entretanto, a Circular n. ${ }^{\circ}$ 3097/DGE/2018 veio revogar a Circular n. ${ }^{\circ} 3 /$ DSEEAS/DGE/2013 (81) apresentando, entre outras alterações, uma maior adequação das capitações dos GA às necessidades energéticas e nutricionais das crianças nas faixas etárias a que se destinam. Embora esta nova Circular mencione que o cálculo das capitações dos GA se baseia no manual "Capitações de géneros alimentícios para refeições em meio escolar: fundamentos, consensos e reflexões" publicado em 2015 (82), apresenta alguns valores não fundamentados para as capitações da DM. De igual modo, para ir ao encontro da Lei n. ${ }^{\circ}$ 11/2017 de 17 de Abril, define capitações dos GA para a opção vegana nas cantinas escolares, mas não menciona a fonte do cálculo das mesmas, sabendo-se também que o manual acima mencionado não as apresenta. Ainda em relação à opção vegana, a nova Circular alerta "para a singularidade destas opções que, se não forem bem planeadas, podem provocar algumas carências e desequilíbrios nutricionais, pelo que se aconselha o máximo cuidado na sua elaboração".

\section{ANÁLISE CRÍTICA}

O direito à alimentação é intransponível e constitui um direito fundamental básico com carácter universal e simultaneamente individual, indivisível, interdependente e inter-relacionado. Além disso, é um direito social por pressupor a intervenção do Estado na garantia das necessidades nutricionais dos indivíduos com alimentos de elevada qualidade nutricional (83). A Assembleia-Geral das Nações Unidas aprovou em 1959 a Declaração dos Direitos da Criança onde é explícito, no princípio 4. ․, "A criança (...) tem direito a crescer e a desenvolver-se com boa saúde (...) a uma adequada alimentação, habitação, recreio e cuidados médicos" (84).

Como demonstrado, não existe consenso científico quanto ao impacto na saúde de uma DV, com posições como a da AND (2016), que indica que a DV é adequada a todas as fases da vida quando bem planeada (35), e a da Associação Alemã de Nutrição, que não recomenda uma dieta vegana na "gravidez, lactação, infância e adolescência", devido a um elevado risco de deficiências nutricionais (40). Em paralelo, sabe-se que são escassos e de baixa qualidade os estudos sobre o impacto da adoção deste tipo de dieta na saúde das crianças e adolescentes (7, 51), levando alguns autores a questionarem a posição da AND (2016) (85). Outro questionamento é que, ao contrário da DM, não existe uma definição formal e objetiva de DV, embora exista uma concordância social que nesta dieta não se consuma carne nem pescado, mas que poderá incluir laticínios e/ou ovos. Em acréscimo, é aceite pela comunidade científica que quanto mais restritiva for a dieta, maior o risco de carência nutricional e por isso, o alerta é maior no caso da DV estrita ou vegana (44). Note-se que contrariando este dado, a opção vegetariana obrigatória nas ementas dos refeitórios escolares portugueses é a DV estrita ou vegana (3). Outro ponto a necessitar de critério científico, é o cálculo das capitações dos GA para a DV, mensuração que em contexto de alimentação coletiva permite uma abordagem técnica às necessidades energéticas e nutricionais. Mais, seria de se equacionar uma revisão das capitações dos GA e dos critérios alimentares da DM presentes na Circular (79), aproximando os seus valores às reais necessidades energéticas e nutricionais da população em idade escolar, e alavancando medidas de sustentabilidade e de combate ao desperdício alimentar, tão características da DM.

Sendo o refeitório escolar um espaço educativo e de prática de hábitos alimentares saudáveis, e estando definida a promoção da DM como um dos Princípios Gerais da Circular n. ${ }^{\circ}$ 3097/DGE/2018, será necessário refletir sobre as questões supra apresentadas e o consequente porquê da obrigatoriedade de se disponibilizarem opções alimentares que não colhem o consenso da comunidade científica quanto ao seu impacto na saúde. Mesmo ponderando-se a DV como uma tendência de consumo alimentar, não é conhecida a sua prevalência em crianças portuguesas (embora este pressuposto não deva suportar uma tomada de decisão técnico-científica, como se devem qualificar as que abrangem a oferta alimentar em meio escolar do estado português).

Por último, é imperioso refletir-se na necessidade de investimento na promoção da DM em meio escolar, de modo planeado, programado e com recursos adequados. De facto, a Lei de Bases do Sistema Educativo nacional expressa a alimentação escolar como uma medida de ação social, com carácter universal, destinada a todos os alunos de modo a assegurar uma alimentação equilibrada e adequada às suas necessidades (86). O conceito de DM enquadra-se nestes objetivos, não só pela sua excelente qualidade nutricional, mas como veículo educacional, cultural e social, pelo que a sua prossecução em contexto escolar deve ser abalizada por nutricionista(s) dos diversos intervenientes sectoriais.

\section{CONCLUSÕES}

O refeitório escolar é um espaço educativo onde se encontra estabelecido por Lei, a promoção, divulgação e defesa da DM, reconhecida como "uma forma de comer promotora da saúde, sustentável e amiga do ambiente".

Neste artigo é abordado o potencial impacto de uma DV, na saúde e na educação de crianças em idade escolar, bem como o seu pretenso nivelamento com a DM.

Poucos ou nenhuns estudos foram feitos sobre o impacto da introdução de uma opção vegana em crianças de idade escolar, nem se apresentou o porquê da necessidade de se criar uma opção à DM. Não será despiciendo recordar que as refeições em contexto escolar são uma competência do Ministério da Educação, que deve garantir a promoção da saúde através da alimentação e não privilegiar opções alimentares que, ao não seguirem os princípios da DM, potenciam o risco nutricional. 
Urge que o Ministério da Educação desenvolva um projeto com vista à efetiva implementação da DM nos refeitórios das escolas portuguesas.

\section{AGRADECIMENTOS}

As autoras manifestam uma nota final de agradecimento à Professora Doutora Cristina Santos, pela sua contribuição na revisão deste artigo.

\section{REFERÊNCIAS BIBLIOGRÁFICAS}

1. Pinho JP, Silva SCG, Borges C, Santos CT, Santos A, Guerra A, et al. Alimentação vegetariana em idade escolar. Lisboa: Direção-Geral da Saúde; 2016. 59 p.

2. Di Genova T, Guyda H. Infants and children consuming atypical diets: Vegetarianism and macrobiotics. Paediatrics \& Child Health. 2007;12(3):185-8.

3. Assembleia da República. Lei n 11/2017 de 17 Abril, (2017).

4. Beardsworth AD, Keil ET. Vegetarianism, Veganism, and Meat Avoidance: Recent Trends and Findings. British Food Journal, MCB UP Ltd. 1991;93(4):19-24.

5. Le LT, Sabate J, Singh PN, Jaceldo-Siegl K. The Design, Development and Evaluation of the Vegetarian Lifestyle Index on Dietary Patterns among Vegetarians and NonVegetarians. Nutrients. 2018;10(5).

6. Phillips F. Vegetarian nutrition. Nutrition Bulletin. 2005;30(2):132-67.

7. Dagnelie PC, Mariotti F. 1 - Vegetarian Diets: Definitions and Pitfalls in Interpreting Literature on Health Effects of Vegetarianism. In: Mariotti F, editor. Vegetarian and Plant-Based Diets in Health and Disease Prevention: Academic Press; 2017. p. 3-10. 8. Leitzmann C. Vegetarian nutrition: past, present, future. Am J Clin Nutr. 2014;100 Suppl 1:496S-502S.

9. Dwyer J. VEGETARIAN DIETS. In: Caballero B, editor. Encyclopedia of Food Sciences and Nutrition (Second Edition). Oxford: Academic Press; 2003. p. 5974-9.

10. Solomons NW. Plant-based diets are traditional in developing countries: 21 st century challenges for better nutrition and health. Asia Pacific Journal of Clinical Nutrition. 2000;9(S1):S41-S54.

11. Ethical vegetarian. (n.d.) Farlex Partner Medical Dictionary 2012 [cited 2019, Julho] Available from: https://medical-dictionary.thefreedictionary.com/ethical+vegetarian. 12. AVP. Associação Vegetariana Portuguesa: O que é o vegetarianismo? [cited 2019, Julho]. Available from: https://www.avp.org.pt/informacao/o-que-e-o-vegetarianismo/. 13. Hoffman SR, Stallings SF, Bessinger RC, Brooks GT. Differences between health and ethical vegetarians. Strength of conviction, nutrition knowledge, dietary restriction, and duration of adherence. Appetite. 2013;65:139-44.

14. Radnitz C, Beezhold B, DiMatteo J. Investigation of lifestyle choices of individuals following a vegan diet for health and ethical reasons. Appetite. 2015;90:31-6.

15. Ruby MB. Vegetarianism. A blossoming field of study. Appetite. 2012;58(1):141-50. 16. Centro Vegetariano. Ética move vegetarianos.2017 [cited 2019, Julho]. Available from: https://www.centrovegetariano.org/index.php?\&article_id=518.

17. Ensaff H, Coan S, Sahota P, Braybrook D, Akter H, McLeod H. Adolescents' Food Choice and the Place of Plant-Based Foods. Nutrients. 2015;7(6).

18. European Vegetarian Union. Statistics on Vegetarian Lifestyles and Products: European Vegetarian Union (EVU); 2019 [cited 2019, Julho]. Available from: https:// www.euroveg.eu/public-affairs/statistics-on-vegetarian-lifestyles-and-products/. 19. Sathyamala C. Meat-eating in India: Whose food, whose politics, and whose rights? Policy Futures in Education. 2018.

20. Biswas S. The myth of the Indian vegetarian nation: BBC news; 2018 [cited 2019 , Julho]. Available from: https://www.bbc.com/news/world-asia-india-43581122.

21. Ministry of Home Affairs Government of India. Sample Registration System Baseline Survey 2014 India: Office of the Registrar General \& Census Commissioner; 2014 [cited 2019, Julho].

22. 14\% da população se declara vegetariana: IBOPE Inteligência; 2018 [cited 2019, Julho]. Available from: http://ibopeinteligencia.com/noticias-e-pesquisas/14-dapopulacao-se-declara-vegetariana/.

23. Morgan R. The slow but steady rise of vegetarianism in Australia.2016 [cited 2019, Julho]. Available from: http://www.roymorgan.com/findings/vegetarianisms-slow-butsteady-rise-in-australia-201608151105.

24. Number of vegans and vegetarians in Germany: Vebu; 2018 [cited 2019, Julho].
Available from: https://vebu.de/veggie-fakten/entwicklung-in-zahlen/anzahl-veganerund-vegetarier-in-deutschland/.

25. Reland J. Are there 3.5 million vegans in the UK? UK: Full Fact; 2018 [cited 2019, Julho]. Available from: https://fullfact.org/health/vegans-uk/.

26. Kay Magistad M. https://www.pri.org/stories/2013-06-27/vegan-lunch-goingmeatless-beijing: PRI's The World; 2013 [cited 2019, Julho]. Available from: https:// www.pri.org/stories/2013-06-27/vegan-lunch-going-meatless-beijing.

27. Stahler C. How many people are vegan? How many eat vegan when eating out? Asks the vegetarian resource group: The Vegetarian Resource Group; 2019 [cited 2019, Julho]. Available from: https://www.vrg.org/nutshell/Polls/2019_adults_veg.htm. 28. Hernando S. The vegan era, or the end of carnal pleasures: El País; 2019 [cited 2019, Julho]. Available from: https://elpais.com/elpais/2019/02/07/ inenglish/1549556881_820642.html.

29. Bandeira M. Número de vegetarianos em Portugal quadruplicou em 10 anos: Jornal Económico; 2018 [cited 2019, Julho]. Available from: https://jornaleconomico.sapo. pt/noticias/numero-de-vegetarianos-em-portugal-quadruplicou-em-10-anos-299824. 30. Mensink GB, Kleiser C, Richter A. [Food consumption of children and adolescents in Germany. Results of the German Health Interview and Examination Survey for Children and Adolescents (KiGGS)]. Bundesgesundheitsblatt, Gesundheitsforschung, Gesundheitsschutz. 2007;50(5-6):609-23.

31. Young Consumers Are Hungry For Meat Alternatives In Germany [press release]. Mintel Press office.2015.

32. Reporter $S$. One in ten British children are refusing to eat meat and identify as vegan or vegetarian, survey finds.: The Sun; 2019 [cited 2019, Julho]. Available from: https:// www.thesun.co.uk/news/9060050/more-british-kids-are-vegan/.

33. Vegetarian Resource Group. How Many Teens And Other Youth Are Vegetarian And Vegan? The Vegetarian Resource Group Asks In A 2014 National Poll: Vegetarian Resource Group (VRG); 2014 [cited 2019, Julho]. Available from: https://www.vrg.org/ blog/2014/05/30/how-many-teens-and-other-youth-are-vegetarian-and-vegan-thevegetarian-resource-group-asks-in-a-2014-national-poll/.

34. Pediatric Nutrition, 7th Edition. Kleinman REGFR, editor: American Academy of Pediatrics; 2013 2013-10-15 00:00:00. 1460 p.

35. Melina V, Craig W, Levin S. Position of the Academy of Nutrition and Dietetics: Vegetarian Diets. Journal of the Academy of Nutrition and Dietetics. 2016;116(12):1970-80.

36. National Health and Medical Research Council. Australian Dietary Guidelines Canberra: National Health and Medical Research Council 2013 [cited 2019, Julho]. Available from: https://www.eatforhealth.gov.au/guidelines.

37. Agnoli C, Baroni L, Bertini I, Ciappellano S, Fabbri A, Papa M, et al. Position paper on vegetarian diets from the working group of the Italian Society of Human Nutrition. Nutrition, Metabolism and Cardiovascular Diseases. 2017;27(12):1037-52.

38. Conselho Regional de Nutricionistas. Parecer Técnico CRN-3 No 11/2015: Vegetarianismo. 2015.

39. Amit M. Vegetarian diets in children and adolescents. Paediatrics \& child health. 2010;15(5):303-14.

40. Richter M, Boeing H, Grünewald-Funk D, Heseker H, Kroke A, Leschik-Bonnet E, et al. Vegan diet. Position of the German Nutrition Society (DGE). German Nutrition Society (DGE). 2016;63(04):92-102. Erratum in: 63(05): M262.

41. WHO. Complementary feeding [Agosto, 2019]. Available from: https://www.who. int/nutrition/topics/complementary_feeding/en/.

42. Fewtrell M, Bronsky J, Campoy C, Domellöf M, Embleton N, Fidler Mis N, et al. Complementary Feeding: A Position Paper by the European Society for Paediatric Gastroenterology, Hepatology, and Nutrition (ESPGHAN) Committee on Nutrition. Journal of Pediatric Gastroenterology and Nutrition. 2017;64(1):119-32.

43. Silva SCG, Pinho JP, Borges C, Santos CT, Santos A, Graça P. Linhas de orientação para uma alimentação vegetariana saudável. Lisboa: Direção-Geral da Saúde; 2015.50 p. 44. Lobato L, Pinho JP, Cramês M, Santos CT, Graça P. Planeamento de refeições vegetarianas para crianças em restauração coletiva: Princípios Base. Lisboa: DireçãoGeral da Saúde; 2016. 46 p.

45. Documentos PNPAS de apoio à implementação de refeições vegetarianas em cantinas públicas: Direção-Geral da Saúde; 2017 [cited 2019, Julho]. Available from: 
https://nutrimento.pt/noticias/documentos-pnpas-de-apoio-a-implementacao-derefeicoes-vegetarianas-em-cantinas-publicas/.

46. Monarca Almeida C. Vegetarianismo. Estas são as nove perguntas mais frequentes, agora com respostas 2019 [cited 2019, Julho]. Available from: http:// www.ordemdosnutricionistas.pt/noticia.php?id=764.

47. Lusa. Nutricionistas com reservas na aplicação de ementa vegetariana nas cantinas públicas: Diário de Notícias; 2017 [cited 2019, Julho]. Available from: https://www. dn.pt/sociedade/interior/nutricionistas-com-reservas-na-aplicacao-de-ementavegetariana-nas-cantinas-publicas-8520459.html.

48. Candeias V, Nunes El, Morais Cl, Cabral M, Ribeiro da Silva P. Princípios para uma Alimentação Saudável. Lisboa: Direção-Geral da Saúde; 2005. 31 p.

49. Baroni L, Goggi S, Battaglino R, Berveglieri M, Fasan I, Filippin D, et al. Vegan Nutrition for Mothers and Children: Practical Tools for Healthcare Providers. Nutrients. 2018;11(1). 50. Louwman MWJ, van Dusseldorp M, van de Vijver FJR, Thomas CMG, Schneede $J$, Ueland PM, et al. Signs of impaired cognitive function in adolescents with marginal cobalamin status. The American Journal of Clinical Nutrition. 2000;72(3):762-9

51. Schurmann S, Kersting M, Alexy U. Vegetarian diets in children: a systematic review. European journal of nutrition. 2017;56(5):1797-817.

52. UNESCO. Cyprus, Croatia, Spain, Greece, Italy, Morocco and Portugal Inscribed in 2013 (8.COM) on the Representative List of the Intangible Cultural Heritage of Humanity 2013 [cited 2019, Julho]. Available from: https://ich.unesco.org/en/decisions/8. $\mathrm{COM} / 8.10$

53. Graça P, Queiroz J, Cabral CB. Manifesto pela preservação da Dieta Mediterrânica 2018 [cited 2019, Julho]. Available from: https://nutrimento.pt/noticias/manifesto-pelapreservacao-da-dieta-mediterranica/.

54. Graça P, Mateus MP, Lima RM. O Conceito de Dieta Mediterrânica e a Promoção da Alimentação Saudável nas Escolas Portuguesas. Revista Nutrícias. 2013:06-9.

55. Serra-Majem L, Trichopoulou A, de la Cruz JN, Cervera P, Álvarez AG, La Vecchia $\mathrm{C}$, et al. Does the definition of the Mediterranean diet need to be updated? Public Health Nutrition. 2007;7(7):927-9.

56. Trichopoulou A, Bamia C, Trichopoulos D. Anatomy of health effects of Mediterranean diet: Greek EPIC prospective cohort study. BMJ. 2009;338:b2337.

57. Pinho I, Rodrigues S, Franchini B, Graça P. Padrão alimentar mediterrânico: promotor de saúde. Lisboa.: Direção-Geral da Saúde,; 2015. 40 p.

58. Dinu M, Pagliai G, Casini A, Sofi F. Mediterranean diet and multiple health outcomes: an umbrella review of meta-analyses of observational studies and randomised trials. European Journal Of Clinical Nutrition. 2017;72:30.

59. Malmir H, Saneei P, Larijani B, Esmaillzadeh A. Adherence to Mediterranean diet in relation to bone mineral density and risk of fracture: a systematic review and metaanalysis of observational studies. European journal of nutrition. 2018;57(6):2147-60. 60. Rafie N, Golpour Hamedani S, Barak F, Safavi SM, Miraghajani M. Dietary patterns, food groups and telomere length: a systematic review of current studies. European Journal Of Clinical Nutrition. 2016;71:151.

61. Tilman D, Clark M. Global diets link environmental sustainability and human health. Nature. 2014;515:518

62. Appleby PN, Crowe FL, Bradbury KE, Travis RC, Key TJ. Mortality in vegetarians and comparable nonvegetarians in the United Kingdom. The American journal of clinical nutrition. 2016;103(1):218-30.

63. Mihrshahi S, Ding D, Gale J, Allman-Farinelli M, Banks E, Bauman AE. Vegetarian diet and all-cause mortality: Evidence from a large population-based Australian cohor - the 45 and Up Study. Preventive Medicine. 2017;97:1-7.

64. Kwok CS, Umar S, Myint PK, Mamas MA, Loke YK. Vegetarian diet, Seventh Day Adventists and risk of cardiovascular mortality: A systematic review and meta-analysis. International Journal of Cardiology. 2014;176(3):680-6.

65. Dinu M, Abbate R, Gensini GF, Casini A, Sofi F. Vegetarian, vegan diets and multiple health outcomes: A systematic review with meta-analysis of observational studies. Critical Reviews in Food Science and Nutrition. 2017;57(17):3640-9.

66. Lopes C, Torres D, Oliveira A, Severo M, Alarcão V, Guiomar S, et al. Inquérito Alimentar Nacional e de Atividade Física, IAN-AF 2015-2016: Relatório de resultados. Universidade do Porto, 2017
67. Gregório MJ, Rodrigues AM, Graça P, de Sousa RD, Dias SS, Branco JC, et al. Food Insecurity Is Associated with Low Adherence to the Mediterranean Diet and Adverse Health Conditions in Portuguese Adults. Frontiers in Public Health. 2018;6(38). 68. Pereira-da-Silva L, Rêgo C, Pietrobelli A. The Diet of Preschool Children in the Mediterranean Countries of the European Union: A Systematic Review. Int J Environ Res Public Health. 2016;13(6):572.

69. Iaccarino Idelson P, Scalfi L, Valerio G. Adherence to the Mediterranean Diet in children and adolescents: A systematic review. Nutrition, Metabolism and Cardiovascular Diseases. 2017;27(4):283-99.

70. D'Innocenzo S, Biagi C, Lanari M. Obesity and the Mediterranean Diet: A Review of Evidence of the Role and Sustainability of the Mediterranean Diet. Nutrients. 2019;11(6):1306

71. Graça P, Gregório MJ. A Construção do Programa Nacional para a Promoção da Alimentação Saudável: Aspectos Conceptuais, Linhas Estratégicas e Desafios Iniciais. Revista Nutrícias. 2013:06-9.

72. Graça P, Gregório M. Evolução da política alimentar e de nutrição em Portugal e suas relações com o contexto internacional. Alimentação Humana. 2012:79-96. 73. World Health Organization. Regional Office for Europe. Food and nutrition policy for schools : a tool for the development of school nutrition programmes in the European Region. Copenhagen: WHO Regional Office for Europe; 2006. Available from: https:// apps.who.int/iris/handle/10665/107797.

74. Commission Of The European Communities. WHITE PAPER ON A Strategy for Europe on Nutrition, Overweight and Obesity related health issues.2007 [cited 2019, Agosto]; 279. Available from: https://ec.europa.eu/health/nutrition_physical_activity/policy/strategy_pt. 75. Ministério da Educação. Decreto-Lei no 55/2009. Diário da República $n^{\circ} 42,1^{\text {a }}$ série de 2009-03-02:1424-33. Estabelece o regime jurídico aplicável à atribuição e ao funcionamento dos apoios no âmbito da acção social escolar, (2009).

76. Ministério da Agricultura, do Desenvolvimento Rural e das Pescas, da Saúde e da Educação. Portaria n 1242/2009. Diário da República nº 197, $1^{a}$ série de 2009-10-12: 7479-82. Aprova o regulamento do regime de fruta escolar., (2009).

77. Ladeiras L, Matias Lima R, Lopes A. Ofício Circular n7/DGE/2013 - Bufetes Escolares - Orientações (revogou Circular n 11/DGIDC/2007). Lisboa: Direção-Geral da Educação; 2012. Available from: http://www.dge.mec.pt/bufetes-escolares.

78. M Isabel Machado Baptista. Educação Alimentar em Meio Escolar - Referencial para uma Oferta Alimentar Saudável. Lisboa: Direcção-Geral de Inovação e de Desenvolvimento Curricular; 2006.

79. Lima RM. Circular n³097/DGE/2018 - Orientações sobre Ementas e Refeitórios Escolares (revogou a Circular n 3/DSEEAS/DGE/ 2013). Lisboa: Direção-Geral da Educação; 2018. 146 p.

80. Associação Vegetariana Portuguesa (AVP). Petição pela inclusão de opções vegetarianas nas escolas, universidades e hospitais portugueses 2016 [cited 2019, Agosto]. Available from: https://peticaopublica.com/pview.aspx?pi=PT76070.

81. Circular $n^{\circ}$ 3/DSEEAS/DGE/ 2013 - Orientações sobre ementas e refeitórios escolares - 2013/2014 (revogou Circulares nº 14 e n 15/DGIDC/2007). Lisboa: Direção-Geral da Educação; 2013. 52 p.

82. Gomes S, Ávila H, Oliveira B, Franchini B. Capitações de géneros alimentícios para refeições em meio escolar: fundamentos, consensos e reflexões. Porto: Associação Portuguesa dos Nutricionistas, Faculdade de Ciências da Nutrição e Alimentação da Universidade do Porto, Programa Nacional para a Promoção da Alimentação Saudável da Direção-Geral da Saúde; 2015.

83. Queiroz A, Mota I, Cardoso S. O Direito à Alimentação Saudável no Contexto das Políticas Nutricionais. Acta Portuguesa de Nutrição. 2015:34-7.

84. Queiroz CA, Soliguetti DFG, Moretti SLdA. As principais dificuldades para vegetarianos se tornarem veganos: um estudo com o consumidor brasileiro. DEMETRA: Alimentação, Nutrição \& Saúde. 2018;13(3):20.

85. Cofnas N. Is vegetarianism healthy for children? Critical Reviews in Food Science and Nutrition. 2019;59(13):2052-60.

86. Ministério da Educação e Ciência - Gabinete do Secretário de Estado do Ensino e da Administração Escolar. Despacho n. ${ }^{\circ} 8452-A / 2015$. Diário da República n. ${ }^{\circ}$ 148/2015, $2^{\circ}$ Suplemento, Série II de 2015-07-31. 